\title{
Research on the Security Based on Web Database
}

\author{
Hua Ren
}

Xi'an Fanyi University, Shaanxi, xi 'an, China, 710105

Keywords: Web System; Database; Security Technology

\begin{abstract}
The rapid development of information technology in the current era, the continuous improvement of information technology level, based on the Web system database technology applications have also been synchronized development. Database technology for enterprises in terms of an important significance, not only related to the economic efficiency of enterprises and development, but also related to the enterprise information security. In the context of network, Web-based database has its own particularity, so its security is more worthy of attention. This paper first analyzes the Web database system, and then analyzes the current security environment facing the Web database, and finally puts forward the technical means of improving the security problem for the security problem.
\end{abstract}

\section{Introduction}

With the continuous development of enterprises in the context of market, information technology in the enterprise application has been continuously promoted, a strong information function for enterprise management has brought great convenience and management efficiency and quality have been improved. The application of the database in the enterprise production, management, management and other aspects are involved, it can be said that any function cannot be separated from the support of database technology. The current era is an era of information technology to occupy the mainstream, and Web-based database is currently widely used, so the study of its security issues is extremely necessary.

\section{The Web Database System Analysis}

Web database through the basic data recording, storage and reading, to achieve a large number of data organization and management, based on the Web system database is to protect the high degree of information sharing, data management more comprehensive and efficient.

Web System Architecture. Web system architecture, including the client, server-side two, this traditional model is also known as C / S mode, because the two layers can be the appropriate task division, so for the entire system, the data communication load can be Effective mitigation. With the development of information technology, C / S architecture model began to appear, in order to improve the system's convenience, scalability and other levels, B / S architecture model has been effectively developed and used, B / S architecture model of the system Resource sharing function is more prominent.

$\mathrm{J} 2 \mathrm{EE}$ architecture is the system architecture model which has absolute superiority in Web system. It mainly includes four layers: client layer, presentation layer, business logic layer and enterprise information system layer. MVC is its implementation mode, and the implementation technology mainly includes JSP + Servlet + JavaBean, SHH framework and so on.

Web Database System. Web system is still the most common application of C / S mode, Web 
system development efficiency, high scalability, high compatibility and other characteristics, making the Web database has been greatly developed. MySQL database is currently the most favored by the enterprise database management system, many advantages to enable it to enterprise information management play a great role. MySQL database is the main feature of small size, powerful, fast response and open source.

\section{Web Database Security Issues Analysis}

System Platform Security Issues. System platform security is the basis of database security, Web system C / S architecture model to make its system vulnerabilities, which also led to the database server vulnerabilities, which for the database information security has brought a greater security threat. For example, due to loopholes caused by unauthorized access activities, will inevitably damage the data and information on the database, a serious security threat. And the enterprise database once in the system platform environment loopholes in the impact of the enterprise information management and will inevitably adversely affect the normal operation.

Audit Function Risk. In the data and information operation of the Web database system, the audit is one of the most important functions. The use of the audit tool is related to the validity and accuracy of the audit. In the Web database system, audit tools often have a series of problems or suspicious phenomena, which will produce security risks, resulting in security threats to audit functions. For example, in the current application of the most widely used Web database system, there are often multiple events at the same time, and this situation is the most prone to suspicious phenomenon, but the current Web database system audit means not Perfect, and thus will inevitably make these suspicious phenomenon into audit risk, so that the security of the database system is affected.

SQL Injection Problem. Database information is related to the important business secrets of the enterprise, and one of the main forms of system intrusion is through the unauthorized access to the database information, in the Web database system, due to SQL channel vulnerability problems make data storage and reading the process is facing security problems. For example, non-normal channel injection of the database statement, after the completion of the SQL injection will have an impact on the entire system, and the implementation of these statements will enable the intruder to have full access to the entire database, resulting in a great damage to the Web database system.

Lack of Complete Authentication Function. Web database system is still not perfect for the current authentication, mainly in the lack of rigor and lack of comprehensive two aspects. Lack of rigor, will make the authentication certificate-related information can easily be stolen, the use of stolen information to $\log$ on the system, seriously affecting system security; lack of comprehensiveness, making the authentication method other than authentication to become a reality, some high-tech Horizontal hackers can log in to the system in an illegal manner and steal authentication information and related credentials.

Refusal of Service. Denial of service is also one of the more common problems in the Web database system. This problem arises because the user is attacked so that the access function cannot be used successfully. Therefore, the denial of service cannot be managed or operated by the user. The denial of service problems, the consequences are often the system crashes, server overload, data corruption and so on. These problems not only have great harm to the database system, but also bring great losses to the enterprise.

Backup Data. Enterprise use of the Web database system backup data storage media, under normal circumstances there is no security measures, which makes the risk of data vulnerabilities increase. Any data in the data storage system is destroyed, it will cause irreparable damage. 


\section{Web Database Security Enhance Technology}

Improve the System Security Model. Strengthening access restrictions and access restrictions is the main way to improve the security level of the system, and it is also a direct technical means to effectively solve the current system platform security problem. In order to ensure the safe operation of the system, establish a sound system security model, we must ensure that the user access to the program and operation, a complete and complete certification. For example, when the user enters the Web database system through the application program, it will go through the authentication steps such as user name and password, and strengthen the system security, and then increase the security when the data is read and stored after the login is completed.

Strengthen the access restrictions to limit the different users of the database information on the operational scope of the object as the main way to achieve. For example, to limit the user's information on the database, the contents of the operation can be, to restrict which users can use what method of operation of information and content and so on.

Strengthen access restrictions by restricting the range of applications that different users have access to the system. For example, to limit which user groups can operate and manage which functional modules of the database, or to restrict user-manageable system workflows.

Strengthen the Audit Trail. In view of the current audit function of Web database system, it is necessary to improve the audit trail. The effective implementation of the audit function, it must be able to play an effective monitoring effect. Therefore, based on the system data, to strengthen the tracking and recording, to timely and effective problems, and tracking, the safety of the database is undoubtedly a very effective protective measure.

Specifically, the first, through the use of audit trail tools, the user behavior in the system to record, generate audit log; second, based on audit trail data to increase the audit alarm function, in order to achieve the system security violations of the automatic response; Third, in the event of an audit alarm, the need for a comprehensive analysis of security violations, and according to the analysis of the results of the authority to adjust to improve the level of system security.

Data Encryption. In the process of application of Web database system, the data risk caused by SQL injection problem is very prominent. In order to effectively deal with the risk of SQL injection problem, the level of data encryption technology is the most important technical means, and the application of encryption technology is more System user access increases security guarantees. For these system function models, a separate set of encryption schemes is established, and this encryption scheme should be implemented in a specific solution. Data encryption system consists of two parts of encryption and decryption and the system administrator cannot directly enter the database, through the decryption calculation before the user function and permissions distribution. And encryption and decryption by the plaintext, encryption algorithm and decryption algorithm, key and other components, data encryption data storage, transmission, will be within the controllable range of the system, which makes the general technology cannot complete the system invasion, To ensure system security.

Improve the Level of Database Server Security Management. Denial of service is one of the main manifestations of database system security problems, which is caused by the lack of security management level, so effectively solve the problem of denial of service and it should improve the level of database server security management. At the same time, the level of security management can also effectively deal with the system authentication function, SQL injection and other issues and improve the overall security level of the database.

Web database in the data acquisition, through business processing, server login and other ways to achieve and improve the level of its safety management, data systems should be based on the data 
function to achieve a reasonable program:

First, protect the timely update procedures, open demand services, disable non-demand services. The timely update of the program is to ensure the premise of security, system service function management, but also related to the probability of attack by the system, for non-demand class service function selection disabled can effectively reduce the possible system attacks.

Second, limit the number of users to limit the number of users. User terminal is a relatively high risk of system security, the limitations of the server terminal, mainly to limit the terminal and the server to connect the scope of the agreement, so as to improve the level of security of the agreement, thus ensuring system security; and the number of users limits increased security for access to the database system.

Finally, run through the NTFS file system control program.

Increase the Differential Backup. Differential backup is one of the most applicable methods in the database backup method. This method is based on the basic data backup function of the database system, and improves the data security after data loss through the backup of the backup system. Differences The contents of the backup include files, database folders, and so on. Each enterprise uses the database function and module is different, but can increase the differential backup to improve the system's risk response ability.

Specifically, the first basic backup function, that is, database backup and backup log two functions to improve, such as database backup need to key system information or data backup, and the need to establish a regular backup program, and backup log You should back up the actual transaction for the system database. Second, the difference between the fixed time interval backup, a specific time for the cycle, regular differential backup, once the problem occurs, you can choose the problem before the last differential backup to restore the data.

\section{Conclusion}

To sum up, Web database security issues exist objectively, and with the enterprise database application is widespread, its security issues have been constantly attached importance to in order to effectively enhance the database security for enterprises to provide greater information management and security, Improve system security Technical management is the inevitable choice for the future. This paper analyzes the different database security technology has a certain feasibility, and easier to implement, but any kind of security technology, network environment, cannot guarantee absolute security, so in the specific application, the need for different security technology to conduct a comprehensive Review and screening, in order to establish their own safety management needs consistent with the database system security technology system.

\section{Acknowledgements}

Fund Project: "Web Programming Course Teaching Team" Project Grant (Project No. Z1312)

\section{References}

[1] Huang Wanyi. E-commerce website database security technology issues[J]. Information Security and Technology, 2012 (04)

[2] Wu Rong. Web database system security management strategy[J]. Computer Security, 2007 (03)

[3] Liu Mengfei. Web database security threats and countermeasures[J]. Computer Knowledge and Technology, 2011 (34) 
[3] ZHU Lian-jun, CUI Qing-hua.Study on Secure Operation Strategy and Related Technology of WEB Database [J]. Journal of Henan Education Institute (Natural Science Edition), 2006 (01)

[5] LUO Ming.Study on security strategy based on WEB database access technology [J]. Science and Technology Innovation Guide, 2009 (32)

[6] Wang Shifeng, Chen Zhigang. Using caching technology to optimize the Web database page performance and security maintenance [J] .Computer \& Information Technology, 2007 (02). 